\title{
BMJ Open Mobile phone text messaging for improving the uptake of vaccinations: a systematic review protocol
}

\author{
Robyn Kalan, ${ }^{1}$ Charles S Wiysonge, ${ }^{2}$ Tshepiso Ramafuthole, ${ }^{1}$ Kurt Allie, ${ }^{1}$ \\ Fatima Ebrahim, ${ }^{1}$ Mark Emmanuel Engel ${ }^{1}$
}

To cite: Kalan $\mathrm{R}$,

Wiysonge CS, Ramafuthole T, et al. Mobile phone text messaging for improving the uptake of vaccinations: a systematic review protocol. BMJ Open 2014;4:e005130. doi:10.1136/bmjopen-2014005130

- Prepublication history for this paper is available online. To view these files please visit the journal online (http://dx.doi.org/10.1136/ bmjopen-2014-005130).

Received 26 February 2014 Revised 23 June 2014 Accepted 25 June 2014

\section{CrossMark}

For numbered affiliations see end of article.

Correspondence to Dr Mark Emmanuel Engel; mark.engel@uct.ac.za

\section{ABSTRACT}

Introduction: Low vaccine coverage is a major public health concern, the consequences of which contribute to around 1.5 million child deaths from vaccinepreventable diseases. Thus, innovative strategies to rapidly increase coverage and recall rates for vaccinations are urgently required. Mobile text messaging (or short messaging service, SMS) has the potential to help increase vaccination coverage and therefore we propose to conduct a review of the current best evidence for the use of SMS as an intervention to promote vaccination coverage.

Methods and analysis: This article describes the protocol for a systematic review of the effectiveness of SMS in improving the uptake of vaccination. Primary and secondary outcomes of interest are prespecified. We will preferably include randomised controlled trials (RCTs). However, non-randomised studies (NRS) will be considered if there is an inadequate number of RCTs. We will search several bibliographic databases (eg,PubMed, EMBASE, CINAHL, CENTRAL, Science Citation Index, Africa-Wide Information and WHOLIS electronic databases and search sources for grey literature. Following data extraction and assessment of risk of bias, we will meta-analyse studies and conduct subgroup analyses, according to intervention subtypes. We will assess clinical heterogeneity and statistical heterogeneity. For outcomes without quantitative data, a descriptive analysis will be used. This review protocol is registered in the PROSPERO International Prospective Register of systematic reviews, registration number 2014:CRD42014007531

Ethics and dissemination: Ethics is not required for this study, given that this is a protocol for a systematic review, which uses published data. The findings of this study will be disseminated through peer-reviewed publications and conference presentations. We anticipate that the results could be used by researchers and policymakers to help inform them of the efficacy of mobile phone text messaging interventions to promote increased vaccination coverage.

Trial registration number: PROSPERO registration number 2014:CRD42014007531.

\section{INTRODUCTION}

Vaccinations, when given at the most sensitive developmental years of childhood, help
Strengths and limitations of this study

To our knowledge, this is the first systematic review protocol that will attempt to assess the impact of mobile text messaging on promoting the uptake of vaccination among adults, adolescents and parents or caregivers of children.

- This study will help inform clinical practice and future studies on the effectiveness of media platforms.

- Non-randomised studies of low-quality evidence may be this study's limitation. We will, however conduct appropriate analyses to assess the overall robustness of the results.

to promote comprehensive and capable immunity, enabling children to fight off certain diseases. ${ }^{1}{ }^{2}$ In addition, vaccinations are widely regarded as one of the most costeffective public health interventions that help to reduce global child morbidity and mortality. ${ }^{3}$ Low coverage of vaccinations is a major public health concern. In Africa alone, more than seven million children did not receive the full spectrum of vaccinations recommended before reaching 1 year of age in $2009 .^{5}$ It is also estimated that 1.5 million children died globally from vaccinepreventable diseases where WHO prequalified vaccines were available. ${ }^{6}$

The Global Vaccine Action plan (GVAP) is the most recently launched global effort by the WHO to help increase vaccination coverage. The GVAP has set a target that by 2020 vaccination coverage for populations should reach $90 \%$ national vaccination coverage and at least $80 \%$ at district levels utilising national vaccination programmes. ${ }^{7}$ It is guided by six principles: country ownership, shared responsibility and partnership, equity, integration, sustainability and innovation. ${ }^{8}$

A variety of factors impact achieving low coverage rates; challenges such as immunisation awareness, demand for immunisation, level of trust in the health system, adequate 
human resources, access, timeliness of vaccinations, service delivery, poor infrastructure and vaccination monitoring. ${ }^{4}$ Vaccination coverage seems to be lower in low-income households, where limited access to health education, contributes to poor health-seeking behaviour along with an inability to improve general well-being. ${ }^{1} 29$ Uneducated parents therefore are less likely to understand the importance of vaccinating to prevent potentially harmful diseases. In light of these obstacles to vaccination coverage, the strategy to improve vaccination coverage needs to be innovative as alluded to in the GVAP, well thought out and able to penetrate low-income households effectively.

Globally, mobile phone use is rapidly increasing, with an estimated six billion mobile phone users worldwide at the end of 2011. ${ }^{10}$ In particular, mobile phone text messaging has gained popularity among people living in low-income and middle-income countries and may be the key to penetrating hard to reach areas in the developing world. Text messaging has proven to be a costeffective method of relaying health information and reminders than the more traditional methods such as face-to-face, phone calls, pamphlets, mail and email. ${ }^{5}$ As immunisation usually requires multiple consecutive monthly visits after the first vaccine dose in order to complete the schedule, short messaging service (SMS) can be used as reminder for an upcoming visit and recall when a visit has been missed. ${ }^{1}$ In addition, an SMS intervention, also known as mobile phone text messaging, can be delivered alone or bundled with other interventions. ${ }^{11}$ Diseases that have used mobile technology successfully include HIV where a $90 \%$ adherence was observed among text message recipients compared with a $40 \%$ adherence in the control group. ${ }^{12}$ We therefore propose to conduct a systematic review of the current best evidence for the use of mobile phone text messaging to improve vaccination coverage.

\section{METHODS}

This review protocol has been published in the PROSPERO International Prospective Register of systematic reviews (http://www.crd.york.ac.uk/PROSPERO), registration number 2014:CRD42014007531. The methods for this review will follow those published previously. ${ }^{13}$

\section{Criteria for considering studies for this review \\ Type of studies}

We will include randomised controlled trials (RCTs), interrupted time series and controlled before and after studies.

\section{Types of participants}

Participants will be caregivers of infants or children, adolescents and adults including pregnant women drawn from any setting, community-based or otherwise.

\section{Types of interventions}

We will include interventions in which mobile phone text messages serve as a reminder to be vaccinated, as educational information or, as information regarding vaccine availability at the clinic in an attempt to promote uptake of vaccinations. Vaccinations could include routine infant immunisations, those against human papilloma virus, influenza, meningococcal or tetanus/diptheria/accellular pertussis. Eligible studies will be those that compared SMS to no intervention, or to other interventions for increasing vaccination coverage. If we find less than 10 studies that include only SMS as the intervention, we will include studies in which mobile phone voice speaking or voice messaging are interventions; studies in which the use of a beeper or pager is the intervention; studies in which the use of multimedia messaging service is the intervention; and studies in which text messages are bundled with other interventions. In such circumstances, we will stratify the analysis by type of intervention.

\section{Types of outcome measures}

Results must include quantitative data for outcomes measured.

\section{Primary outcomes}

The primary outcome is vaccination coverage, irrespective of disease. We will use the definition of vaccine coverage as stipulated by the respective authors.

\section{Secondary outcomes}

Secondary outcomes are the recall rate in persons who had previously missed their vaccinations, scheduled appointments for vaccination or completeness of vaccination records.

\section{Search methods for identification of studies}

A comprehensive and exhaustive search of databases and conference proceedings will be performed by RK with the help of the University of Cape Town librarian, to identify all relevant studies available by 30 June 2014, regardless of language or publication status. We will search both peer-reviewed journal articles and grey literature (unpublished, internal or non-reviewed papers and reports).

\section{Database}

We will search the following electronic databases: PubMed; EMBASE; Cochrane Central Register of Controlled Trials (CENTRAL); ISI Web of Science (Science Citation Index); Africa-Wide Information, Cumulative Index of Nursing and Allied Health (CINAHL) and WHO library databases (WHOLIS). We will use both text words and medical subject heading (MeSH) terms; for example vaccination*, immunization*, immunisation*, "Immunization"(MeSH), "Vaccination"(MeSH), "Immunization, Secondary"(MeSH) OR "Immunization Programs"(MeSH), "Immunization Schedule"(MeSH), "Mass Vaccination"(MeSH), mobile 
phone, text messaging, text*, SMS, reminder*, recall, telemedicine, mHealth and eHealth. These terms will be used in varying combinations. The literature search strategy will be adapted to suit each database. Table 1 shows the main search strategy we will use.

\section{Conference proceedings}

We will search the following conference proceedings for relevant abstracts: Vaccine and International Society for Vaccines Congress, International African Vaccinology Conference, Annual Vaccines Congress, Annual Conference on Vaccine Research, World Congress on Pediatric Infectious Diseases, International Pediatric Association Conference, National Immunization Conference and the Annual Infectious Diseases in Children Symposium.

\section{Searching other sources}

For ongoing studies, we will search the WHO International Clinical trials Registry Platform, Clinicaltrials.gov, Pan African Clinical Trials Registry (PACTR), and contact individual researchers working in the field as well as the following organisations: WHO, Global Alliance for Vaccines and Immunisation, Center for Disease Control and Prevention and mHealth Alliance. We will also search the website of mHealth Alliance and mHealth in the Low Resource Settings resources database for eligible studies.

\section{Reference lists}

We will obtain reference lists of relevant studies identified and the full-text articles reviewed for inclusion in the review will be checked for additional information.

\section{Data collection and analysis}

The methods for data collection and analysis will be based on the Cochrane Handbook of Systematic Reviews for Interventions. ${ }^{14}$

\section{Selection of studies for inclusion}

We will construct a screening guide to ensure that the inclusion criteria are adhered to and consistently applied by all review authors. Two review authors (RK

\begin{tabular}{|c|c|}
\hline Search & PubMed \\
\hline \#1 & $\begin{array}{l}\text { (immunization[Mesh]) OR ((immunis* OR } \\
\text { immuniz* OR vaccin*) }\end{array}$ \\
\hline \#2 & (adolescents OR children OR teenagers) \\
\hline \#3 & $\begin{array}{l}\text { 'SMS' OR cellphone OR 'mobile phone' OR 'text } \\
\text { messaging' OR 'short message service' OR 'text } \\
\text { reminder' }\end{array}$ \\
\hline \#4 & \#1 AND \#2 \\
\hline \#5 & \#3 AND \#4 \\
\hline
\end{tabular}

and MEE), working independently, will screen the titles and abstracts of all studies identified through the literature searches for eligibility. RK will obtain the full text of studies deemed potentially eligible. The two authors (RK and MEE) will independently assess the full text of each article for eligibility, and compare their results and resolve discrepancies by discussion and consensus, consulting a third author (CSW) to resolve any persistent disagreements. For all studies excluded by the assessors we will describe the reasons for exclusion.

\section{Assessment of risk of bias in included studies}

Two authors will independently assess the risk of bias in the included studies. Separate criteria will be used to assess RCTs and non-randomised studies. The criteria used to assess the risk of bias of in RCTs will be random sequence generation; allocation concealment; blinding of participants, study personnel; blinding of outcome assessors; incomplete outcome data; selective outcome reporting; other sources of bias and overall risk of bias, in accordance with the methods used by the Cochrane Collaboration ${ }^{14}$ as well as the Cochrane Consumers and Communication Review Group. ${ }^{15}$ The criteria used for risk of bias assessment for non-randomised studies will include selection bias (with regard to comparability of groups, confounding and adjustment); performance bias (in terms of the fidelity of the interventions and quality of the information regarding who received which interventions, including blinding of study participants and healthcare providers); detection bias (regarding unbiased and correct assessment of outcomes, including blinding of assessors); attrition bias (with regard to completeness of sample, follow-up and data); and reporting bias (with regard to publication biases and selective reporting of results). ${ }^{14}$ Studies will be scored as having low, high or unclear risk of bias. The two authors will resolve disagreements in the assessment of risk of bias by discussion and consensus, consulting a third author to resolve any persistent disagreements.

\section{Data extraction and management}

References will be managed using Thomson ISI Research-Soft Endnote V.9.0. ${ }^{16}$ Two authors will independently extract descriptive and outcome data for each included article using a standardised data collection form, resolving any discrepancies by discussion and consensus; failing which, a third author (CSW) will arbitrate. RK will enter the final data into the Cochrane Collaboration Review Manager V.5.1 statistical software (http://ims.cochrane.org/RevMan). CW will crosscheck the data entered to ensure that there are no data entry errors.

\section{Data synthesis including assessment of heterogeneity}

Data analysis will be conducted using the Cochrane Collaboration Review Manager V.5.1 statistical software (http://ims.cochrane.org/RevMan). The outcomes of interest will be either dichotomous or continuous. We 
will calculate risk ratios and their corresponding 95\% CIs and $\mathrm{p}$ values for dichotomous outcomes, and mean differences and SDs for continuous outcomes. Where outcomes are measured using different scales, we will report standardised mean differences (SMD) ${ }^{17}$ In cases of missing or incomplete information presented in the included studies, we shall contact authors for further information.

We will assess clinical heterogeneity by examining types of participants, interventions and outcomes in each study. We will pool data only from studies judged to be clinically homogenous. Statistical heterogeneity in each meta-analysis will be assessed using the $\chi^{2}$ test and quantified using the $\mathrm{I}^{2}$ statistic. ${ }^{18}$ If studies are sufficiently homogenous (in terms of study populations, interventions and outcomes), then we will pool the data across studies and estimate summary effect sizes using a fixed-effects model. Otherwise, we will use the random-effects model. Should heterogeneity remain significant, we will discuss the findings as a narrative summary.

We will perform subgroup analyses by intervention subtypes: long versus short messages; daily versus weekly messages; short weekly messages versus long weekly messages; short daily messages versus long daily messages; and two-way interactive communication versus one-way communication. ${ }^{12} 1920$ We will also stratify analysis by study design (randomised controlled separate from nonrandomised studies) and intervention type (multiple interventions involving text messaging separate from text messaging alone). We will also conduct a subgroup comparison of self-reported vaccination completion versus verified clinic records as well as according to age categories and country setting.

Finally, we will use the grading of recommendations assessment, development and evaluation (GRADE) approach $^{21}$ to assess the quality of evidence for the effectiveness of the SMS intervention. This method results in an assessment of the quality of the body of evidence as high, moderate, low or very low. Evidence is considered of high quality if 'further research is very unlikely to change our confidence in the estimate of effect'; and moderate quality if 'further research is likely to have an important impact on our confidence in the estimate of effect and may change the estimate'. Low quality evidence implies that 'further research is very likely to have an important impact on our confidence in the estimate of effect and is likely to change the estimate', and very low quality that 'we have very little confidence in the effect estimate'.

\section{Sensitivity analyses}

Several sensitivity analyses will be performed: first to determine whether the study design (RCT vs nonrandomised study) could influence the results of the meta-analysis; second, to evaluate whether the model of the statistical method (random-effects vs fixed-effects model) could change the results and third, to determine the impact of excluding studies with a highrisk bias on the results, with emphasis on allocation concealment, blinded outcome assessment and losses to follow-up (with a cut-off of $25 \%$ loss to follow-up).

\section{Reporting of this review}

Findings in our systematic review will be presented in several ways. Flow diagrams will be used to summarise the study selection process. Funnel plots will be used to assess publication bias if we identify 10 or more eligible studies. The $\kappa$ statistic $^{22}$ will be used to assess agreements between the full-text screening, data extraction and risk of bias assessment by the two authors (RK and MEE). GRADE summary of tables of findings, risk of bias tables or graphs and forest plots will also be used where appropriate. The reporting of outcomes without quantitative data will be descriptive. Lastly, we will provide a list of excluded studies with reasons for exclusion.

\section{Ethics and dissemination}

Systematic reviews draw on publicly available data and do not directly involve human participants, and therefore do not require formal ethical review. ${ }^{23}$ The study protocol will be reviewed by supervisors with expertise in methodology (systematic review) and submitted to the University of Cape Town Departmental Research Committee for approval.

The findings of this systematic review will have implications for policy, practice and research. We will discuss the relevance of our findings to childhood immunisation programmes in Africa in the decade of vaccines with emphasis on applicability, effects on equity, cost implications and monitoring and evaluation. Our systematic review will provide evidence of whether policymakers can adopt mobile phone text messaging alone or in combination with other interventions in efforts to improve uptake of vaccines in national immunisation programmes. It will also inform clinic or hospital managers of how best to use the intervention to improve vaccination coverage. The systematic review may also identify specific considerations that would need to be taken into account for future studies, such as study location, content and timing of messages, whether or not parents or caregivers replied to text messages, how text messages were sent (automated vs manual), indicators for immunisation programmes, variety of text messages sent (inclusion of jokes or lifestyle tips), duration of the study, whether or not participants were provided with the mobile handsets and sample size. ${ }^{24}$

\section{Author affiliations}

${ }^{1}$ Department of Medicine, Faculty of Health Sciences, University of Cape Town, Cape Town, South Africa

${ }^{2}$ Centre for Evidence-based Health Care \& Division of Community Health, Faculty of Medicine and Health Sciences, Stellenbosch University, Cape Town, South Africa 
Acknowledgements The authors would like to acknowledge the critical input and support of the Evidence-Based Medicine Research Support Unit, Faculty of Health Sciences, University of Cape Town.

Contributors CSW, TR, KA and FE conceived of the review. RK and MEE undertook the drafting of the manuscript. RK and MEE analysed the data and participated in the interpretation of the results. All authors developed the design of the protocol were involved in data acquisition and have given their approval for publication.

Competing interests None.

Provenance and peer review Not commissioned; externally peer reviewed.

Open Access This is an Open Access article distributed in accordance with the Creative Commons Attribution Non Commercial (CC BY-NC 3.0) license, which permits others to distribute, remix, adapt, build upon this work noncommercially, and license their derivative works on different terms, provided the original work is properly cited and the use is non-commercial. See: http:// creativecommons.org/licenses/by-nc/3.0/

\section{REFERENCES}

1. Garde M. Inequalities in child survival: looking at wealth and other socio-economic disparities in developing countries. 2010. http:// wwwsavethechildrenorguk/sites/default/files/docs/Final draft inequalities_of_child_survival_10_August_formatted_2_1pdf (accessed 5 Aug 2012).

2. Multiple Authors. Vaccines \& preventable diseases. National Center for Immunization and Respiratory Disease. 2009 http://wwwcdcgov/ vaccines/vpd-vac/defaulthtm

3. Bloom DE. The value of vaccination. Adv Exp Med Biol 2011;697:1-8.

4. Machingaidze S, Wiysonge CS, Hussey GD. Strengthening the expanded programme on immunization in Africa: looking beyond 2015. PLoS Med 2013;10:e1001405.

5. Stockwell MS, Kharbanda EO, Martinez RA, et al. Text4Health: impact of text message reminder-recalls for pediatric and adolescent immunizations. Am J Public Health 2012;102:e15-21.

6. World Health Organisation and UNICEF: Global Immunization Data. http://wwwwhoint/immunization_monitoring/Global_Immunization_ Datapdf (accessed 10 Jun 2013).

7. WHO Global Vaccination Action Plan. http://wwwwhoint/immunization monitoring/Global_Immunization_Datapdf (accessed 10 Jun 2013).

8. Decade of Vaccines (DoV) Collaboration: Draft global vaccine action plan. http://wwwdovcollaborationorg/action-plan/ (ccessed 10 Jun 2013)
9. Kaewkungwal J, Singhasivanon P, Khamsiriwatchara A, et al. Application of smart phone in "Better Border Healthcare Program": a module for mother and child care. BMC Med Inform Decis Mak 2010;10:69.

10. Roter DL, Hall JA, Merisca R, et al. Effectiveness of interventions to improve patient compliance: a meta-analysis. Med Care 1998;36:1138-61.

11. Mohammed S, Siddiqi O, Ali O, et al. User engagement with and attitudes towards an interactive SMS reminder system for patients with tuberculosis. J Telemed Telecare 2012;18:404-8.

12. Pop-Eleches $\mathrm{C}$, Thirumurthy $\mathrm{H}$, Habyarimana JP, et al. Mobile phone technologies improve adherence to antiretroviral treatment in a resource-limited setting: a randomized controlled trial of text message reminders. AIDS 2011;25:825-34.

13. Nglazi MD, Bekker LG, Wood R, et al. Mobile phone text messaging for promoting adherence to anti-tuberculosis treatment: a systematic review. BMC Infect Dis 2013;13:566.

14. Higgins JPT, Green S, eds. Cochrane Handbook for Systematic Reviews of Interventions. Version 5.0.2 [updated September 2009]. The Cochrane Collaboration, 2009. http://www.cochrane-handbook. org. 2009

15. Ryan R HS, Prictor M, McKenzie J. Cochrane Consumers and Communication Review Group. Study Quality Guide. 2011. http:// cccrgcochraneorg/sites/cccrgcochraneorg/files/uploads/ StudyQualityGuide May2011pdf (accessed 01 February 2013)

16. Thomson. http://www.endnote.com. ISI Research-Soft Endnote 90

17. Borenstein M. Introduction to meta-analysis. Chichester, UK: John Wiley \& Sons, 2009.

18. Higgins JP, Thompson SG. Quantifying heterogeneity in a meta-analysis. Stat Med 2002;21:1539-58.

19. Horvath T, Azman H, Kennedy GE, et al. Mobile phone text messaging for promoting adherence to antiretroviral therapy in patients with HIV infection. Cochrane Database Syst Rev 2012;3. CD009756.

20. Thirumurthy $\mathrm{H}$, Lester RT. M-health for health behaviour change in resource-limited settings: applications to HIV care and beyond. Bull World Health Organ 2012;90:390-2.

21. Balshem $H$, Helfand $M$, Schunemann $H J$, et al. GRADE guidelines: 3. Rating the quality of evidence. J Clin Epidemiol 2011:64:401-6.

22. Landis JR, Koch GG. The measurement of observer agreement for categorical data. Biometrics 1977;33:159-74.

23. Emanuel EJ, Wendler D, Killen J, et al. What makes clinica research in developing countries ethical? The benchmarks of ethical research. J Infect Dis 2004;189:930-7.

24. Karanja S, Mbuagbaw L, Ritvo P, et al. A workshop report on HIV mHealth synergy and strategy meeting to review emerging evidence-based mHealth interventions and develop a framework for scale-up of these interventions. Pan Afr Med J 2011;10:37. 